\title{
Utility of PET/CT in the staging and treatment of patients with Merkel Cell Carcinoma and Melanoma
}

\author{
Pavlos Papavasiliou*, Rodrigo Arrangoiz and Jeffrey M. Farma
}

Department of Surgical Oncology, Fox Chase Cancer Center, Philadelphia, Pennsylvania, USA

\begin{abstract}
Cutaneous malignancies constitute some of the most commonly diagnosed cancers in the United States (USA), comprising more than half of all cancers diagnosed per year [1]. In this review article we aim to present our experience at a tertiary referral center using PET/CT in the staging and treatment planning in patients with Merkel cell carcinoma (MCC) and melanoma and review the current literature.
\end{abstract}

\section{Introduction}

Merkel cell carcinoma (MCC) is a rare primary cutaneous neuroendocrine malignancy that generally occurs in sun damaged skin of Caucasians, with an increased incidence in older patients. The incidence of MCC in the United States has recently been reported to have increased by an estimated annual percentage change of $8 \%$ from 1986 to 2001 (35), and approximately 1500 new cases were predicted by the American cancer society in the United States alone in 2008 (36). MCC is one of the few cancers found to be associated with immune suppression and polyomavirus has been shown to be integrated within the genome [2]. MCC is an aggressive tumor with an overall five-year survival of $40 \%$ [3]. Reported five year survival rates of local, nodal and metastatic disease are $64 \%, 39 \%$ and $18 \%$, respectively [3]. Reported rates of regional lymph node involvement at the time of presentation vary from 10 to $45 \%$ and around $50 \%$ of patients with lymph node metastases harbor concurrent distant metastases, most often in the liver, lung, brain, bone or skin.

Cutaneous melanoma represents approximately four percent of all skin cancers diagnosed each year, but accounts for approximately $75 \%$ of skin cancer related deaths. In the USA in the year 2010, 68,130 new cases of melanoma were diagnosed, with 8,700 patients dying of the disease [4]. The incidence has been increasing dramatically. The age-adjusted incidence of melanoma in the United States increased from approximately four per 100,000 to 18 per 100,000 in white males between 1973 and 1998, and was reported as over 20 per 100,000 as of $2006[4,5]$. The incidence of cutaneous melanoma continues to increase significantly, increasing in men more rapidly than any other malignancy and, in women more rapidly than any other malignancy except lung cancer [6]. The lifetime risk of developing melanoma for someone born in the USA in the year 2000 is 1 in 41 for men and 1 in 61 for women [5]. Accurate staging and follow-up in melanoma patients is essential for appropriate treatment planning and may improve survival $[7,8]$. High-risk melanoma patients have a recurrence and mortality risk higher than $35 \%$ to $50 \%$ within five years $[7,9]$.

Due to the drastic differences in survival based on initial staging and the presence of nodal or distant metastases, accurate staging modalities are crucial in providing information that would alter treatment planning for patients with these aggressive cutaneous malignancies. Patients identified with distant metastases early in their treatment course could be spared the morbidity associated with operative intervention and proceed with systemic chemotherapy and/ or radiation.

\section{PET/CT}

18-Fluorodeoxyglucose (FDG) positron emission tomography/ computed tomography (PET/CT) is a useful functional imaging modality for the staging of cancer and has a potential role in assessing response to therapy. PET/CT utilizes the increased metabolism of glucose in malignant viable cells. FDG is one of the most commonly used radioisotopes, which is an analogue of glucose and detects the difference in glucose metabolism. Like glucose, FDG is carried into tumor cells, by means of the glucose transporter protein, and is subsequently phosphorylated by a hexokinase to FDG-6-phosphate. FDG-6-phosphate is not a substrate for glucose-6-phosphate isomerase; thus, it is biochemically trapped within the cells. FDG is limited by its inability to differentiate carcinoma from inflammation and research is being performed testing other radiotracers which may improve sensitivity and specificity of the functional imaging. PET/CT plays a role in diagnosis, staging and/or restaging of patients with a variety of different cancers including colorectal, esophageal, head and neck, non-small cell lung cancer, lymphoma, and melanoma [10-12]. Recent studies have found value in preoperative planning for patients with pancreatic and prostate cancer as well $[13,14]$. PET/CT generally can detect lesions approximately $8 \mathrm{~mm}$ in diameter in most commonly used scanners.

\section{PET/CT and Merkel cell carcinoma}

The role and experience with PET/CT in the treatment of MCC is limited in the literature, especially in the preoperative setting. The aggressive nature of MCC suggests that more extensive preoperative evaluation with PET/CT may alter the definitive surgical treatment plan in these complex patients. Accurate preoperative staging in patients with MCC helps to identify patients with advanced disease that would not benefit from invasive local treatments. Identifying unexpected distant disease prior to initiating operative therapy is crucial in the

*Corresponding author: Pavlos Papavasiliou, Department of Surgical Oncology, Fox Chase Cancer Center, Philadelphia, Pennsylvania, USA, E-mail: Pavlos.Papavasiliou@fccc.edu

Received December 13, 2011; Accepted January 25, 2012; Published January 27, 2012

Citation: Papavasiliou P, Arrangoiz R, Farma JM (2011) Utility of PET/CT in the staging and treatment of patients with Merkel Cell Carcinoma and Melanoma. $J$ Nucl Med Radiat Ther S5:001. doi:10.4172/2155-9619.S5-001

Copyright: @ 2012 Papavasiliou P, et al. This is an open-access article distributed under the terms of the Creative Commons Attribution License, which permits unrestricted use, distribution, and reproduction in any medium, provided the original author and source are credited. 
multidisciplinary care of these patients, and spares unwarranted morbidity of an operation, which ultimately would not affect the course of their disease.

Concannon et al. has published one of the largest studies examining the role of PET/CT in the management of MCC. In this retrospective review of 18 patients, common indications for PET/CT scanning included suspected nodal disease or distant metastases at the time of diagnosis. Based on the PET/CT results, $43 \%$ of patients had an alteration in ultimate treatment. This included addition or withdrawal of radiotherapy, surgery or chemotherapy, or an alteration in radiotherapy fields or more extensive operative resections. In their series, 4 of 9 patients were upstaged, and one patient was downstaged. The sensitivity for proven disease was $94 \%$ and $100 \%$ for all lesions greater than $5 \mathrm{~mm}$. Two patients were diagnosed with second malignancies of different histopathology as a result of PET/CT findings (basal cell and salivary gland malignancy [15].

Iagaru et al. also conducted a retrospective review of 6 patients with MCC who underwent a total of $12 \mathrm{PET} / \mathrm{CT}$ scans. Nine true positive lesions, seven true negative lesions, one false positive lesion and one false negative lesion was identified out of the $12 \mathrm{PET} / \mathrm{CT}$ scans. Although statistical analysis could not be performed in this small patient cohort, the authors concluded that PET/CT may have a role in initial staging and post-therapy surveillance [16].

The authors have conducted a retrospective review of their own experience using PET/CT in the preoperative setting in patients with MCC (37). Overall, alteration in management occurred in $44 \%$ of our patients as a result of PET/CT findings. The majority of these findings (73.3\%) included unanticipated identification of metastases to either lymph nodes or distant sites significantly altering treatment course. The remaining patients whose management was altered occurred secondary to either incidental cancers (thyroid, carcinoid) or benign findings (colonic adenoma, inflammatory hilar lymph node) requiring additional testing.

PET/CT identified distant metastases in $15 \%$ of the patients. Sensitivity and negative predictive value were $100 \%$ for detecting metastases. Lower values for the specificity and positive predictive value occurred because preoperative PET/CT identified two second primary tumors as an incidental finding, which were initially felt to be metastases.

While this study did evaluate the role of PET/CT in detecting lymph node metastases from MCC, it has been shown that PET/CT is not accurate in detecting micrometastases for other cancers. The technique requires a minimal volume of disease for cancer to be detected on PET/ CT $[17,18]$. A sensitivity of $73.6 \%$ for detecting lymph node metastases was found in this study, further affirming the benefit of sentinel lymph node biopsy to identify micrometastases within lymph nodes. Nonetheless, PET/CT avid lymph nodes will likely harbor cancer, while lymph nodes that are not PET/CT avid could still potentially harbor micrometastic disease.

Our current algorithm for evaluating patients with MCC includes a preoperative $\mathrm{PET} / \mathrm{CT}$ on all patients. If the $\mathrm{PET} / \mathrm{CT}$ is negative patients are taken for a radical excision of their lesion with sentinel lymph node biopsy, followed by adjuvant radiation. If PET/CT demonstrates concern for hypermetabolic lymph nodes, an ultrasound guided biopsy is used to confirm disease and patients then undergo radical resection and complete lymphadenectomy of the affected lymph node basin, followed by adjuvant radiation to both sites and consideration for chemotherapy (Figure 1,2).

\section{PET/CT and melanoma}

Wahl et al. [19] and Kern [20] demonstrated that radiolabeled glucose analogs were preferentially taken up in murine and human melanoma xenografts, setting forth the rationale for the potential use of using the FDG tracer in patients with melanoma. PET/CT is not useful in the initial staging of thin and intermediate thickness primary cutaneous melanoma when there is no clinical evidence of local or distant metastatic spread. The reason for this is the small size/volume of most nodal metastases combined with the low prevalence of nodal and distant disease in patients with early primary melanomas [21] Friedman et al. [21], recommend using PET/CT for evaluating patients with cutaneous melanoma who fit into one of the four following categories: 1) individuals with a high risk for distant metastases based on extent of loco-regional disease, 2) patients with findings that are suspicious for distant metastases, 3) individuals with known distant tumor deposits who still stand to benefit from customized therapies if new lesions are discovered or treated lesions regress, and 4) patients at high risk for systemic relapse who are considering aggressive medical therapy.

The staging of patients with melanoma is a crucial step in the sequence of events that leads to instituting appropriate management. Again multiple studies have shown that PET/CT has no role in the initial staging of thin and intermediate thickness melanomas in the absence of signs or symptoms suggestive of distant disease [22-24]. One of the first articles to suggest that PET/CT was not as sensitive for staging of regional nodes in patients with cutaneous melanomas came from a prospective trial containing 70 patients with primary melanomas (> $1.0 \mathrm{~mm}$ in thickness) and four patients with recurrent melanoma who underwent PET/CT and sentinel lymph node (SLN) biopsy. The biopsy results were used as a gold standard for regional lymph node metastases. PET/CT scans found two true positives, 71 true negatives, 0 false positives, and 16 false negatives for a sensitivity of $11 \%$ and specificity of $100 \%$. In this study Wagner et al [24], concluded that PET/CT is an insensitive indicator of occult regional lymph node metastases in patients with melanoma because of the minute tumor volumes in this population.

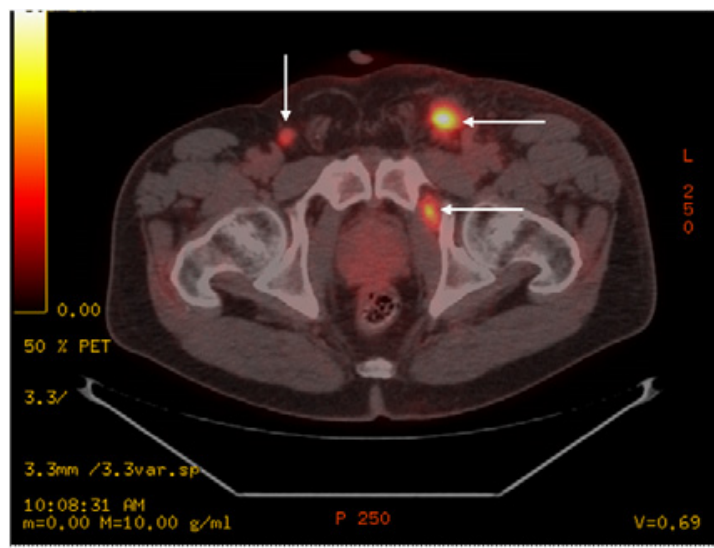

Figure 1. Preoperative PET/CT findings from a 67 year old man with a left buttock primary Merkel cell carcinoma. This study confirmed the presence of palpable left inguinal lymph node metastases (horizontal white arrow) in addition to left obturator lymph node avidity, but also demonstrated avid right inguinal lymphadenopathy (vertical white arrow) that was not clinically palpable. Biopsy of the right inguinal lymph node confirmed the presence of metastatic Merkel cell carcinoma which led to a formal right inguinal lymphadenectomy. 
Citation: Papavasiliou P, Arrangoiz R, Farma JM (2011) Utility of PET/CT in the staging and treatment of patients with Merkel Cell Carcinoma and Melanoma. J Nucl Med Radiat Ther S5:001. doi:10.4172/2155-9619.S5-001
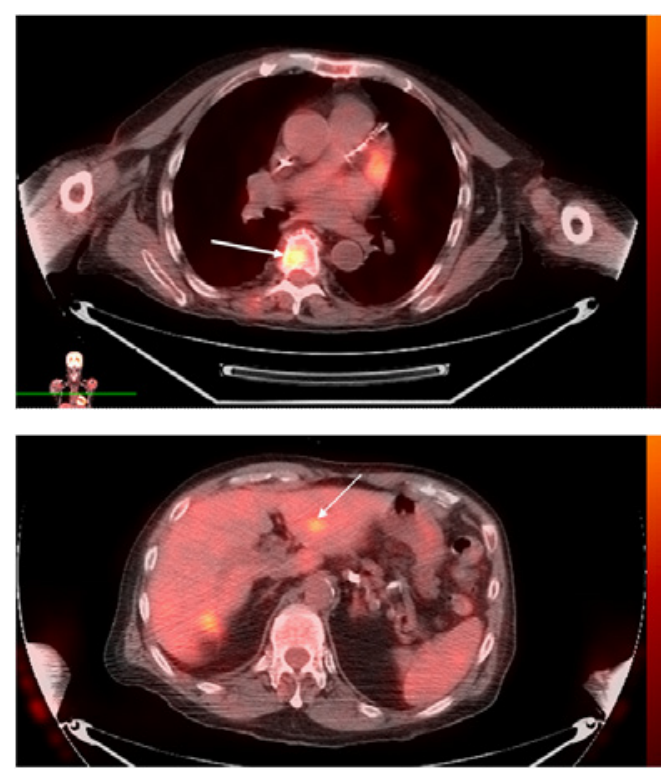

Figure 2. Preoperative PET/CT of a 75 year old man with a Merkel cell carcinoma of the right ear. PET/CT findings include vertebral and liver metastases (figure 2) and right periocular lymph node avidity (not included in images). The patient was initiated on palliative chemotherapy.

In another study, Belhocine et al. showed that of six histologically positive SLNs in patients with clinically localized disease, PET/CT identified only one metastatic focus in a SLN that was greater than one centimeter. Acland et al. [25], found that in 50 patients who underwent SLN biopsy for melanomas that were pathologically greater than 1 $\mathrm{mm}$ in thickness or invading lymphatic's, PET/CT failed to identify all 14 positive SLNs. In another study of 609 patients staged using PET/ CT (pooled from several studies), 38 patients (6\%) had abnormal uptake outside the primary site or regional nodes, but only 1 had a true melanoma metastasis [26]. Blessing et al. [27], in a study of 20 patients utilized ultrasonography and PET to assess regional lymph node involvement (a total of 83 lymph nodded were assessed with these modalities). PET revealed a sensitivity of $74 \%$ and a specificity of $93 \%$.

The diagnostic yield of preoperative PET/CT for regional and distant metastases [28-32] for thin and intermediate thickness melanoma is low. A previous study from our institution retrospectively reviewed 83 patients with intermediate thickness melanoma who underwent SLN biopsy, of which $45 \%$ had preoperative PET/CT. Only two patients from this study with positive SLNB were found to have avid lymph nodes on preoperative PET/CT. This study did not support the routine use of PET/CT for patients undergoing SLNB for melanoma [18].

In a study from our institution evaluating patients with high risk T4 melanomas that were staged preoperatively with PET/CT, 12 (21\%) patients had regional metastatic disease and four (7\%) patients had distant metastatic disease that was identified with preoperative PET/CT (38). Our study showed that the sensitivity and specificity of PET/CT for identifying regional metastatic disease was $40 \%$ and $90 \%$ respectively. In the literature the sensitivity and specificity of PET/ CT for identifying regional metastatic disease has varied from $8 \%$ to $100 \%$ and $84 \%$ to $100 \%$ respectively. The sensitivity and specificity of PET/CT for identifying distant metastatic disease in the literature has ranged from $78 \%$ to $100 \%$, and $22 \%$ to $87 \%$ respectively [28-32]. In our study, the sensitivity and specificity for identifying distant metastatic disease was $100 \%$ and $94 \%$ respectively. PET/CT findings did contribute important information that led to the modification of the original treatment plan and discovered an incidental finding in 20 cases that required further evaluation.

$\mathrm{PET} / \mathrm{CT}$ can affect the treatment planning for melanoma in various ways. In a retrospective cohort of 100 scans, PET/CT showed an added value over and above conventional imaging modalities by upstaging in ten cases, down staging in 24 cases, and depiction of more lesions within the same stage of disease in 15 cases [33]. Damian et al. [34], reported that PET/CT findings influenced the therapeutic management in $22 \%(22 / 100)$ of the patients. In addition, PET/CT was used to clarify another 12 cases where CT was inconclusive. Similarly, Tyler et al (31) and Acland et al. [30] reported the change in management in 15\% and $28 \%$ of patients with PET/CT.

It is the authors practice to obtain PET/CT imaging in melanoma patients with T4 lesions prior to radical excision. Other melanoma lesions evaluated with SLNB (presence of mitosis, lesions thicker than $0.75 \mathrm{~mm}$, and ulcerated lesions) undergo PET/CT imaging after radical excision and SLNB if the SLNB is positive. If the PET/CT does not identify distant metastases, patients are advised to undergo lymphadenectomy of the affected basin (Figure 3,4).

\section{Conclusions}

The reported utility of PET/CT in patients with MCC and high risk melanoma in the literature is limited. However, our studies, as well as, others support the use of PET/CT for staging both high risk melanoma patients and all MCC patients in the pretreatment setting. It

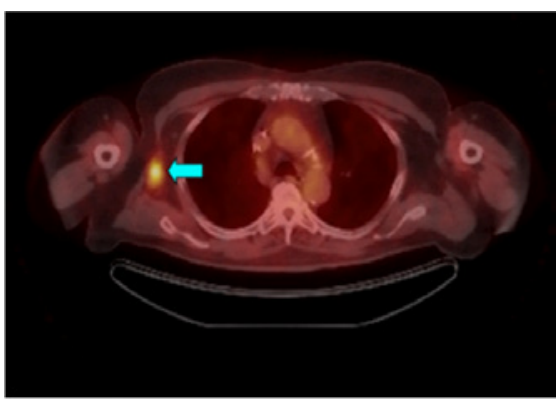

Figure 3: PET/CT showing a positive right axillary lymph node uptake for known right shoulder melanoma.

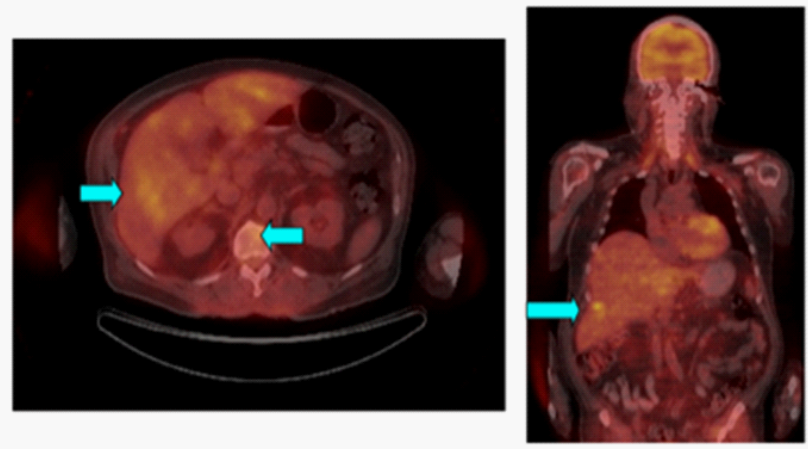

Figure 4: PET/CT showing positive uptake in the liver and vertebra, consistent with metastatic disease from known back melanoma. 
Citation: Papavasiliou P, Arrangoiz R, Farma JM (2011) Utility of PET/CT in the staging and treatment of patients with Merkel Cell Carcinoma and Melanoma. J Nucl Med Radiat Ther S5:001. doi:10.4172/2155-9619.S5-001

is the author's recommendation that all patients diagnosed with MCC or high risk T4 or Stage III melanomas undergo PET/CT imaging at initial diagnosis, as a significant portion of these patients will have an alteration in staging or treatment planning.

\section{References}

1. Christenson LJ, Borrowman TA, Vachon CM, Tollefson MM, Otley CC, et al. (2005) Incidence of basal cell and squamous cell carcinomas in a population younger than 40 years. JAMA 294: 681-690.

2. Schowalter RM, Pastrana DV, Pumphrey KA, Moyer AL, Buck CB (2010) Merkel cell polyomavirus and two previously unknown polyomaviruses are chronically shed from human skin. Cell Host Microbe 7: 509-515.

3. Lemos BD, Storer BE, Iyer JG, Phillips JL, Bichakjian CK, et al. (2010) Pathologic nodal evaluation improves prognostic accuracy in Merkel cell carcinoma: analysis of 5823 cases as the basis of the first consensus staging system. J Am Acad Dermatol 63: 751-761.

4. Jemal A, Siegel R, Ward E, Hao Y, Xu J, et al. (2009) Cancer statistics. CA Cancer J Clin 59: 225-249.

5. Greenlee RT, Hill-Harmon MB, Murray T, Thun M (2001) Cancer statistics. CA Cancer J Clin 51: 15-36.

6. Desmond RA, Soong SJ (2003) Epidemiology of malignant melanoma. Surg Clin North Am 83: 1-29.

7. Balch CM, Gershenwald JE, Soong SJ, Thompson JF, Atkins MB, et al. (2009) Final version of 2009 AJCC melanoma staging and classification. J Clin Oncol 27: 6199-6206.

8. Dummer R, Hauschild A, Pentheroudakis G (2009) Cutaneous malignant melanoma: ESMO clinical recommendations for diagnosis, treatment and follow-up. Ann Oncol Suppl 4: 129-131.

9. Gershenwald JE, Thompson W, Mansfield PF, Lee JE, Colome MI, et al. (1999) Multi-institutional melanoma lymphatic mapping experience: the prognostic value of sentinel lymph node status in 612 stage I or II melanoma patients. J Clin Oncol 17: 976-983.

10. Dietlein M, Weber W, Schwaiger M, Schicha H (2003) [18F-Fluorodeoxyglucose positron emission tomography in restaging of colorectal cancer]. Nuklearmedizin 42: $145-156$

11. Larson SM, Nehmeh SA, Erdi YE, Humm JL (2005) PET/CT in non-small-cel lung cancer: value of respiratory-gated PET. Chang Gung Med J 28: 306-314.

12. Ell PJ (2006) The contribution of PET/CT to improved patient management. Br J Radiol 79: 32-36.

13. Farma JM, Santillan AA, Melis M, Walters J, Belinc D, et al. (2008) PET/CT fusion scan enhances CT staging in patients with pancreatic neoplasms. Ann Surg Oncol 15: 2465-2471.

14. Schmid DT, John H, Zweifel R, Cservenyak T, Westera G, et al. (2005) Fluorocholine PET/CT in patients with prostate cancer: initial experience. Radiology 235: 623-628.

15. Concannon R, Larcos GS, Veness M (2010) The impact of (18)F-FDG PET-CT scanning for staging and management of Merkel cell carcinoma: results from Westmead Hospital, Sydney, Australia. J Am Acad Dermatol 62: 76-84.

16. lagaru A, Quon A, McDougall IR, Gambhir SS (2006) Merkel cell carcinoma: Is there a role for 2-deoxy-2-[f-18]fluoro-D-glucose-positron emission tomography/ computed tomography? Mol Imaging Biol 8: 212-217.

17. Wagner JD, Schauwecker DS, Davidson D, Wenck S, Jung SH, et al. (2001) FDG-PET sensitivity for melanoma lymph node metastases is dependent on tumor volume. J Surg Oncol 77: 237-242.

18. Kell MR, Ridge JA, Joseph N, Sigurdson ER (2007) PET CT imaging in patients undergoing sentinel node biopsy for melanoma. Eur J Surg Oncol 33: 911-913.

19. Wahl RL, Hutchins GD, Buchsbaum DJ, Liebert M, Grossman HB, et al. (1991) 18F-2-deoxy-2-fluoro-D-glucose uptake into human tumor xenografts
Feasibility studies for cancer imaging with positron-emission tomography Cancer 67: 1544-1550.

20. Kern KA (1991) [14C]deoxyglucose uptake and imaging in malignan melanoma. J Surg Res 50: 643-647.

21. Friedman KP, Wahl RL (2004) Clinical use of positron emission tomography in the management of cutaneous melanoma. Semin Nucl Med 34: 242-253.

22. Miranda EP, Gertner M, Wall J, Grace E, Kashani-Sabet M, et al. (2004) Routine imaging of asymptomatic melanoma patients with metastasis to sentinel lymph nodes rarely identifies systemic disease. Arch Surg 139: 831-836; discussion 836-837.

23. Clark PB, Soo V, Kraas J, Shen P, Levine EA (2006) Futility of fluorodeoxyglucose $F 18$ positron emission tomography in initial evaluation of patients with T2 to T4 melanoma. Arch Surg 141: 284-288.

24. Wagner JD, Schauwecker D, Davidson D, Coleman JJ 3rd, Saxman S, et al. (1999) Prospective study of fluorodeoxyglucose-positron emission tomography imaging of lymph node basins in melanoma patients undergoing sentinel node biopsy. J Clin Oncol 17: 1508-1515.

25. Acland KM, Healy C, Calonje E, O'Doherty M, Nunan T, et al. (2001) Comparison of positron emission tomography scanning and sentinel node biopsy in the detection of micrometastases of primary cutaneous malignan melanoma. J Clin Oncol 19: 2674-2678.

26. Ho Shon IA, Chung DK, Saw RP, Thompson JF (2008) Imaging in cutaneous melanoma. Nucl Med Commun 29: 847-876.

27. Blessing C, Feine U, Geiger L, Carl M, Rassner G, et al. (1995) Positron emission tomography and ultrasonography. A comparative retrospective study assessing the diagnostic validity in lymph node metastases of malignan melanoma. Arch Dermatol 131: 1394-1398.

28. Finkelstein SE, Carrasquillo JA, Hoffman JM, Galen B, Choyke P, et al. (2004) A prospective analysis of positron emission tomography and conventional imaging for detection of stage IV metastatic melanoma in patients undergoing metastasectomy. Ann Surg Oncol 11: 731-738.

29. Swetter SM, Carroll LA, Johnson DL, Segall GM (2002) Positron emission tomography is superior to computed tomography for metastatic detection in melanoma patients. Ann Surg Oncol 9: 646-653.

30. Acland KM, O'Doherty MJ, Russell-Jones R (2000) The value of positron emission tomography scanning in the detection of subclinical metastatic melanoma. J Am Acad Dermatol 42: 606-611.

31. Tyler DS, Onaitis M, Kherani A, Hata A, Nicholson E, et al. (2000) Positron emission tomography scanning in malignant melanoma. Cancer 89: 1019 1025.

32. Eigtved A, Andersson AP, Dahlstrom K, Rabol A, Jensen M, et al. (2000) Use of fluorine-18 fluorodeoxyglucose positron emission tomography in the detection of silent metastases from malignant melanoma. Eur J Nucl Med 27: 70-75.

33. Stas M, Stroobants S, Dupont P, Gysen M, Hoe LV, et al. (2002) 18-FDG PET scan in the staging of recurrent melanoma: additional value and therapeutic impact. Melanoma Res 12: 479-490.

34. Damian DL, Fulham MJ, Thompson E, Thompson JF (1996) Positron emission tomography in the detection and management of metastatic melanoma. Melanoma Res 6: 325-329.

35. Hodgson NC (2005) Merkel cell carcinoma: changing incidence trends. J Surg Oncol 89: 1-4.

36. Becker JC (2010) Merkel cell carcinoma. Ann Oncol 21: 81-85.

37. Papavasiliou P, Arrangoiz R, Turaka A, Lango M, Yu JQ, et al. (2011) Preoperative positron emission tomography/computed tomography changes management in patients with Merkel cell carcinoma. Poster presentation, Society of Surgical Oncology Annual Cancer Symposium, March 2011.

38. Arrangoiz R, Papavasiliou P, Kairys J, Mastrangelo MJ, Berger AC, et al. (2011) Preoperative FDG-PET/CT (PET) is an important tool in the management of patients with thick (T4) melanoma. Poster presentation, Society of Surgical Oncology Meeting, March 2011.
This article was originally published in a special issue, Nuclear Medicine in Radiation Oncology handled by Editor(s). Dr. Aruna Turaka, Fox Chase Cancer Center, USA 